\title{
Characterization of High Temperature Mechanical Properties Using Laser Ultrasound
}

\section{International High Power Laser Ablation Conference}

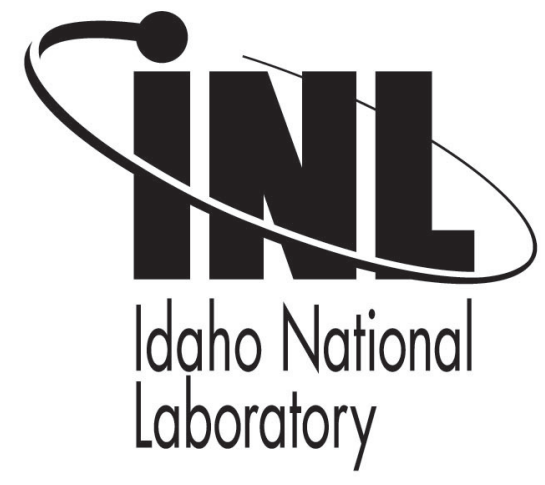

\author{
David H. Hurley \\ Stephen J. Reese \\ Rory Kennedy \\ Farhad Farzbod \\ Richard N. Wright
}

May 2012

This is a preprint of a paper intended for publication in a journal or proceedings. Since changes may be made before publication, this preprint should not be cited or reproduced without permission of the author. This document was prepared as an account of work sponsored by an agency of the United States Government. Neither the United States Government nor any agency thereof, or any of their employees, makes any warranty, expressed or implied, or assumes any legal liability or responsibility for any third party's use, or the results of such use, of any information, apparatus, product or process disclosed in this report, or represents that its use by such third party would not infringe privately owned rights. The views expressed in this paper are not necessarily those of the United States Government or the sponsoring agency. 


\title{
Characterization of High Temperature Mechanical Properties Using Laser Ultrasound
}

\author{
David H. Hurley ${ }^{1}$, Stephen J. Reese ${ }^{1}$, Rory Kennedy ${ }^{2}$, Farhad Farzbod ${ }^{1}$, \\ Richard N. Wright ${ }^{1}$ \\ ${ }^{I}$ Department of Materials Science and Engineering \\ ${ }^{2}$ Department of Fundamental Fuels Properties \\ Idaho National Laboratory, P.O. Box 1625, Idaho Falls, Idaho 83415-2209, USA
}

\begin{abstract}
Laser ultrasound is employed to assess high temperature mechanical properties. A pulsed laser is used for broadband ultrasound generation, and a laser interferometer is used to detect ultrasonic vibrations. For this investigation both time and frequency domain studies are conducted. For the time domain study we measure the modulus of elasticity and the shear modulus of a nickel alloy up to $1000^{\circ} \mathrm{C}$. For the frequency domain study we measure ultrasonic attenuation of a rolled copper sample during high temperature annealing. It is shown that attenuation is associated with grain alignment during the recrystallization transformation.
\end{abstract}

Keywords: Laser ultrasound, grain microstructure, high temperature, mechanical properties. PACS: 62.20.dj, 62.20.de, 62.80.+f, 81.40.Jj, 81.40.Ef

\section{INTRODUCTION}

Mechanical properties are controlled by the evolution of defect structures such as dislocations, vacancies, interstitials, and grain boundaries. All of these microstructural features involve a perturbation of the perfect lattice (i.e. strain fields). Viewed in this context, high frequency strain waves (i.e. ultrasound) provide a natural choice to study mechanical properties. Ultrasonic techniques have long been used to assess changes in the microstructure of industrial materials. For example, techniques have been developed to monitor acoustic attenuation due to thermal aging embrittlement [1], residual stress [2], and changes in texture [3,4]. Traditionally, ultrasound has been generated and detected using piezoelectric transducers. The requirement of intimate contact between the transducer and the material of interest precludes this approach from use in high temperature applications. In addition, the necessity for acoustically matched contact complicates attenuation measurements.

Laser ultrasonics, with optical acoustic generation and detection, has unique advantages over other ultrasonic methods: it is non contacting, requires no couplant or invasive sample preparation (other than that used in metallurgical analysis), and has the demonstrated capability to probe microstructure on a micron scale [5]. As a consequence, laser techniques are highly reproducible, enabling sophisticated data analysis that reveals the state of material microstructure [6]. Generation of ultrasound is accomplished by irradiating a specimen with a short pulse (100 ps $-10 \mathrm{~ns})$ from a 
high intensity laser. A portion of the incident energy is absorbed, giving rise to a rapid, localized thermal expansion of the sample (thermoelastic generation is dominant in metals), which in turn generates ultrasonic waves that propagate through the material. Complimenting ultrasonic generation, laser interferometry is used to detect ultrasonic waves. Because light is being used for generation and detection of the ultrasonic wave, the specimen being examined is not mechanically coupled to the transducer. As a result, laser ultrasound can be carried out remotely, an especially attractive characteristic for in situ measurements of material properties in high temperature environments.

Laser ultrasound studies can be carried out in the time domain or the frequency domain. Time domain studies are well suited to measure the arrival of both longitudinal and shear ultrasonic waves. This information is used to define the elastic stiffness tensor. Complimentary information regarding acoustic attenuation can be obtained by conducing frequency domain studies. This approach involves investigation of the resonant response of a sample with known geometry and dimensions. In this manuscript we report on two laser ultrasonic studies to measure mechanical properties of materials in high temperatures. In the first study we use time domain laser ultrasound to measure the elastic constants of a nickel alloy up to $1000^{\circ} \mathrm{C}$. In the second study we use frequency domain laser ultrasound to measure changes in grain morphology of a pure copper sample during high temperature annealing.

\section{EXPERIMENT}

The experimental setup used for ultrasonic generation and detection is shown in Fig. 1. This approach has been described in detail previously [7] and is only briefly discussed here. Broadband resonant ultrasonic modes are thermoelastically excited in a sample using a pulsed neodymium-doped yttrium aluminum garnet (Nd:YAG) laser operating at $1064 \mathrm{~nm}$ with a pulse duration of $10 \mathrm{~ns}$. A photorefractive interferometer operating at $532 \mathrm{~nm}$ is used to detect out of plane surface motion associated with the resonant modes of vibration. For high temperature investigations the generation and detection laser beams irradiate opposite sides of a sample residing inside a tube furnace. The sample is maintained in a nitrogen environment to keep the surface from oxidizing.

\section{Samples}

For our time domain study we consider a nickel alloy designated Inconel 617. This material is a solid-solution, strengthened nickel-chromium-cobalt-molybdenum alloy used for high temperature structural applications. The sample geometry is that of a flat disc with a thickness of $2.55 \mathrm{~mm}$ and a diameter of $12.70 \mathrm{~mm}$. The generation beam is focused to a tight spot $(\sim 50 \mu \mathrm{m})$ near the center of the sample and bulk waves are detected along the epicentral axis on the opposite side of the sample.

For our frequency domain study, the sample is a high purity copper rectangular parallelepiped. To ensure that we excite as many modes as possible, the 


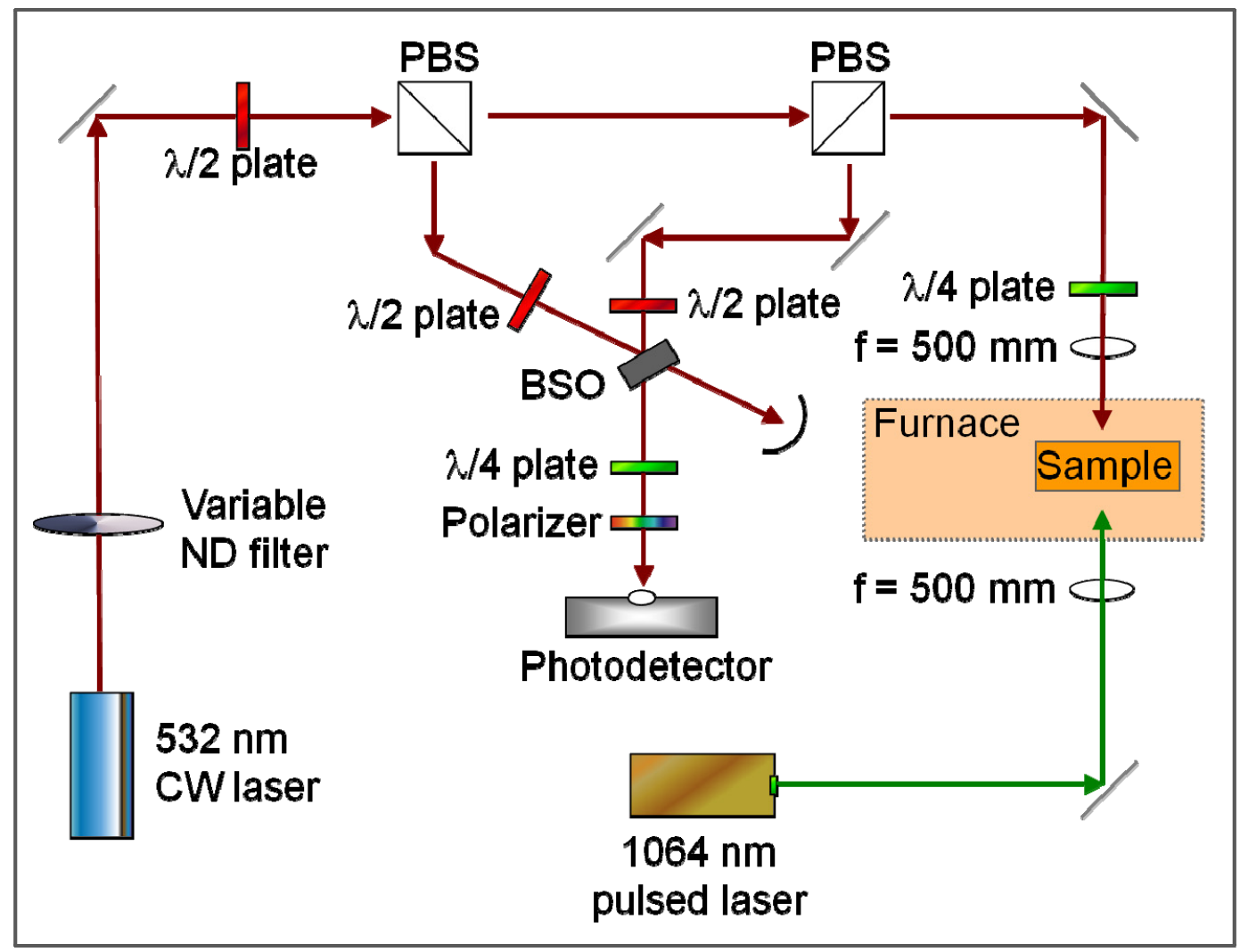

FIGURE 1. Experimental setup. A pulsed Nd:YAG laser is used for ultrasonic excitation, and a photorefractive interferometer is used to detect out of plane motion associated with ultrasonic waves. A furnace purged with nitrogen and equipped with windows for optical access is used to heat the sample.

generation beam strikes a low symmetry location near the corner of the sample. The sample is support by three acoustically mismatched ceramic balls in a specially designed sample holder. The sample has a textured microstructure imparted by rolling a fully annealed material to a $97.5 \%$ reduction. The sample dimensions are $5.40 \times 3.36$ $\times 0.52 \mathrm{~mm}$.

\section{TIME DOMAIN RESULTS}

A typical waveform for detection along the epicentral axis in Inconel 617 is shown in the left side of Fig. 2. This data, taken at room temperature, is used as a baseline for our high temperature studies. The first wave arrival (peak) at approximately $460 \mathrm{~ns}$ corresponds to the longitudinal wave and the second (trough) at approximately $820 \mathrm{~ns}$ corresponds to the shear wave. Using the sample density, the wave arrival times can be converted into elastic moduli. At room temperature these values are $E=200 \mathrm{GPa}$, and $G=78 \mathrm{GPa}$. This analysis assumes that the elastic properties of the sample are isotropic. To confirm this supposition we compare our experimental results to theory [6]. The theoretical results are superposed on top of the experimental results in the left 
pane of Fig. 2. The close comparison of the two waveforms confirms that the material is elastically isotropic.

Next we conducted the same experiment inside the tube furnace at temperatures up to $1000^{\circ} \mathrm{C}$. The results of this study are compared with vendor data in the right side of Fig. 2. The values for modulus of elasticity, E, and the shear modulus, G, measured in this study are consistently lower than the values provided by the vendor[8]. Also it is noted that our results have considerable scatter in comparison to the vendor data. Because it is unclear exactly how the vendor data were obtained, a detailed comparison is not possible. We can speculate that this discrepancy might be due to measurement error associated with walking of the laser beam caused by optical propagation across large thermal gradients. Beam walking of either the generation or detection laser will result in the actual transit distance being larger than the thickness of the sample. A longer transit distance will result in lower values of both the elastic and shear moduli. This discrepancy could also be due to slight differences in the microstructure of the two samples. Future work will be directed at identifying the source of the scatter in our data.
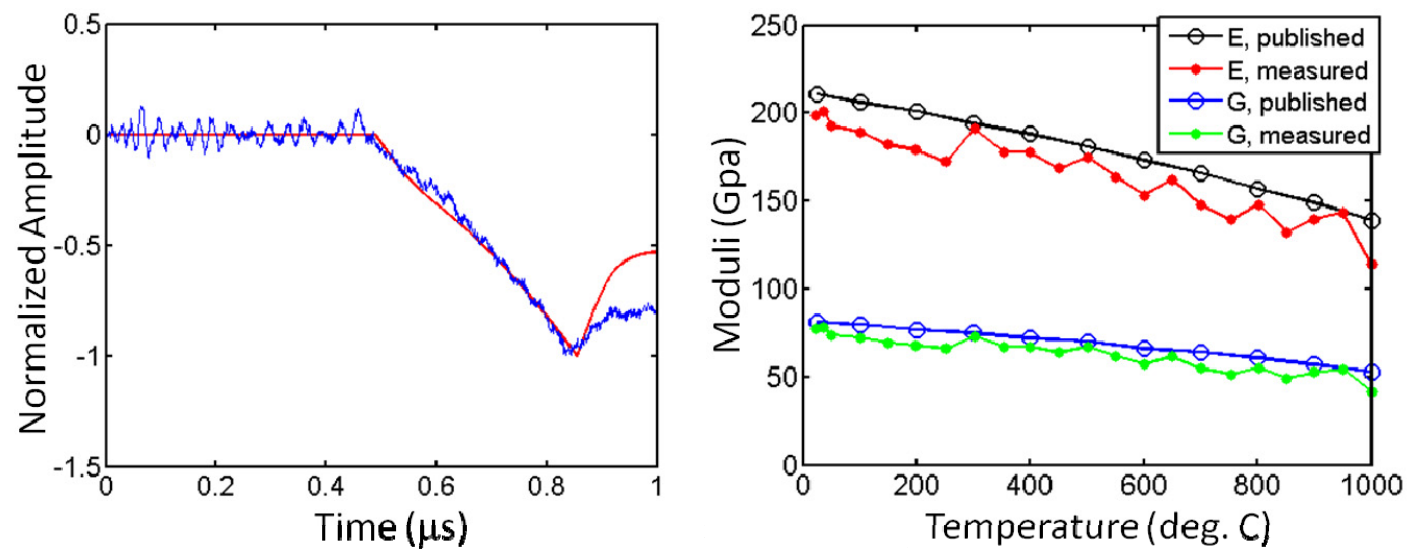

FIGURE 2. Experimental result for time domain study. Left: Comparison between theory and experiment for epicentral waveform. Right: Measured elastic constants up to $1000^{\circ} \mathrm{C}$. These results compare favorably with published values.

\section{FREQUENCY DOMAIN RESULTS}

A typical ultrasonic ringdown waveform used for frequency domain analysis is shown in the left side of Fig. 3. In this case the resonant ultrasound spectrum is obtained by taking the Fourier transform of the ultrasonic ringdown (inset). Using this approach, termed laser resonant ultrasound spectroscopy (LRUS), we can clearly identify resonant modes. Differentiating individual modes is essential for tracking the evolution of grain microstructure. LRUS as it relates to the measurement of elastic constants in a textured material has been described in detail in a previous paper [7]. Here we relate changes in grain morphology upon high temperature annealing to changes in ultrasonic attenuation. The frequency domain approach is ideally suited to measure 

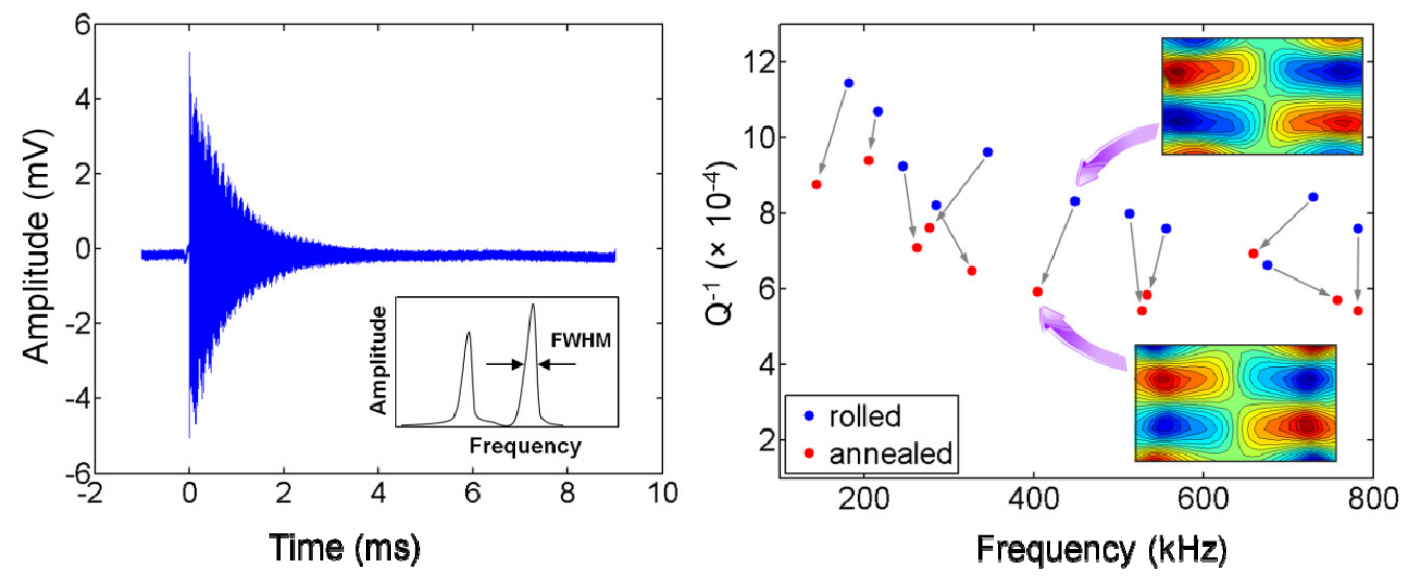

FIGURE 3. Left: Ultrasonic ringdown waveform. The inset shows a portion of the Fourier amplitude spectrum. Right: Measured internal friction values for specific modes before and after annealing. The experimental mode shapes were used to track the internal friction of a particular mode.

ultrasonic attenuation. To understand this point it is convenient to decompose experimental measurements into two categories. In the time domain approach, the amplitude of an ultrasonic pulse is measured at two spatially distinct locations. To measure attenuation, this method requires an accurate accounting of geometric spreading, a computationally intensive approach predicated on having an ultrasound source geometry that is well defined. For the frequency domain approach, attenuation is estimated by measuring the inverse of the full width half maximum (FWHM) of an individual resonant peak $\left(\mathrm{Q}^{-1}\right)$. This approach is insensitive to geometric spreading but does suffer from coupling of ultrasonic energy to the environment. It has been shown [9] that the contactless nature of LRUS minimizes coupling to the environment by employing a sample holder made of a material that is acoustically mismatched to the sample material.

There are many sources of ultrasonic attenuation (e.g. Akheiser, dislocation damping). In this manuscript we address the contribution of grain boundary scattering. The grain boundary scattering mechanism is perhaps more subtle and less studied than other forms of attenuation, which is unfortunate as this contribution to $\mathrm{Q}^{-1}$ can be sizable. Moreover, neglecting the influence of grain boundary scatter can lead to poorly supported conclusions about microstructure mediated changes in $\mathrm{Q}^{-1}$. The physical mechanism relating grain boundary scatter to changes in $\mathrm{Q}^{-1}$ can be understood by noting that a scattered wave, originating from scattering centers having a random size distribution, adds incoherently to the main standing wave. In this case there is no conversion of mechanical energy into heat. In a study involving SiC fiber reinforced Ti composite samples, Ogi et al. [10] show that the ultrasonic attenuation of longitudinal waves is comparable with, or larger than, that of shear waves. This picture is inconsistent with dislocation damping where shear waves more effectively cause dislocations to oscillate about their pinning points. The authors attributed this inconsistency to the contribution to $\mathrm{Q}^{-1}$ from grain boundary scatter.

Grain boundary scatter is caused by a discontinuity in the acoustic impedance across a grain boundary. For materials like copper that have large single crystal 
anisotropy, grain boundary scatter can be significant. For the mode frequencies and grain sizes considered, the scattering of ultrasonic waves at grain boundaries is governed by a power law relationship (Rayleigh regime). In the right side of Fig. 3, the attenuation, $\mathrm{Q}^{-1}$, of eleven peaks before and after annealing are compared. The mode shapes were used to associate the change in attenuation with a particular mode. In all cases the attenuation decreases after annealing. This observation is in keeping with a reduction in damping due to a decrease in dislocation density. However, for polycrystalline materials with large single crystal anisotropy, relating changes in attenuation to changes in microstructure is complicated by attenuation associated with grain boundary scatter [11]. Stanke and Kino [12] have considered grain boundary scatter as a function of average grain size in cubic, non-textured polycrystals. More recently, Yang et al.[13] developed a model for ultrasonic attenuation in a textured hcp polycrystal. As expected, this model demonstrates that, as the polycrystal transforms from randomly aligned (macroscopically isotropic) to perfectly aligned (single crystal), the attenuation due to grain boundary scatter tends to zero.

For the current case, the rolled sample is only lightly anisotropic, having an anisotropy factor close to unity $(A=0.8)$. This value indicates that on average there is a considerable orientation mismatch between adjacent crystallites. The corresponding acoustic impedance mismatch will be large, resulting in considerable grain boundary scatter. Conversely, the annealed sample has an anisotropy factor $(A=2.4)$ closer to the single crystal value $(A=3.2)$. This value indicates that on average the crystallites are highly aligned. In this case both the acoustic impedance mismatch and grain boundary scatter will be small. Thus, as the sample undergoes recrystallization, a reduction in acoustic attenuation is due to both a decrease in dislocation density and a decrease in grain boundary scatter.

Separating these two sources of attenuation will require model development that accounts for the evolving microstructure. It has already been shown[8] that electron backscatter diffraction (EBD) data can be used to gauge the relative importance of texture evolution on the shift in mode frequency. This method suggests an analogous approach to assess the relative contribution of grain boundary scatter. Using a similar approach to that developed by Yang et al. [13] for ultrasonic attenuation, the EBD data could be used to account for the change in attenuation due to grain boundary scatter. This information in turn could be used to gauge the relative significance of grain boundary scatter and dislocation damping.

\section{CONCLUSION}

Time domain laser ultrasound was used to monitor the temperature dependent elastic constants of an Inconel 617 sample up to $1000^{\circ} \mathrm{C}$. The results compare favorably with vendor data indicating the efficacy of laser ultrasound to measure mechanical properties at elevated temperatures. For our frequency domain study we used LRUS to monitor changes in the microstructure mediated mechanical properties during annealing of a high purity copper sample. A decrease in ultrasonic attenuation was observed as the sample passed through the recrystallization temperature. This decrease is attributed to a reduction in ultrasonic attenuation due to reductions in grain boundary scatter and dislocation density. 


\section{ACKNOWLEDGMENTS}

This work was sponsored by the U.S. Department of Energy Fuel Cycle Research and Development Program, under DOE Idaho Operations Office Contract DE-AC0705 ID 14517.

\section{REFERENCES}

1. M. Blaszkiewicz, Nondestructive Characterization of Materials VII, Part 1, 9-15, eds. A. L. Batros, R. E. Green and C. O. Ruud (Transtec Pub. Ltd., Lebanon, New Hampshire, 1996).

2. T. Berruti and M. M. Gola, Nondestructive Characterization of Materials VII, Part 1, 171-178, eds. A. L. Batros, R. E. Green and C. O. Ruud (Transtec Pub. Ltd., Lebanon, New Hampshire, 1996).

3. E. Schneider and L. Oesterlein, Nondestructive Characterization of Materials VII, Part 1, 405-410, eds. A. L. Batros, R. E. Green and C. O. Ruud (Transtec Pub. Ltd., Lebanon, New Hampshire, 1996).

4. G. A. Ahlers, P. Purtschner, J. F. Breedis and F. N. Mandigo, Nondestructive Characterization of Materials VIII, 189-195, ed. R. E. Green (Plenum press, New York, 1998).

5. D. H. Hurley, O. B. Wright, O. Matsuda, T. Suzuki, S. Tamura and Y. Sugawara, Phys. Rev. B 73, 125403 (2006).

6. D. H. Hurley and J. B. Spicer, J. Appl. Phys. 86, 3423 (1999).

7. D. H. Hurley, S. J. Reese, S. Park, Z. Utegulov, J. R. Kennedy, K. Telschow, J. Appl. Phys., 107, 063510 (2010).

8. Vendor data for Inconel 617 elastic moduli provided by Special Metals Corp. Data available at: http://www.specialmetals.com/documents/Inconel alloy 617.pdf.

9. S. J. Reese, K. L. Telschow, T. M. Lillo, and D. H. Hurley, IEEE Trans. Ultrason. Ferroelectr. Freq. Control 55, 770 (2008).

10. H. Ogi, K. Takashima, H. Ledbetter, M. L. Dunn, G. Shimoike, M. Hirao, P. Bowen, Acta mater., 47, 2787 (1999).

11.D. Nicoletti, H. Bilgutay, and B. Onaral, J. Acoust. Soc. Am. 913278 (1992).

12. F.E. Stanke and G. S. Kino, J. Acoust. Soc. Am. 75, 665 (1984).

13. L. Yang, J.A. Turner, and Z. Li, J. Acoust. Soc. Am. 121, 50 (2007). 